\title{
Variations of the celiac trunk and its branches associated with the shift of vascular hilum (porta hepatis) of the liver
}

\author{
Gorantla VR ${ }^{1}$, Nayak $\mathrm{BS}^{2}$, Potu $\mathrm{BK}^{3}$ \\ Department of Anatomy, Kasturba Medical College International Center, Manipal University, Manipal, India. \\ nayaksathish@yahoo.com
}

\begin{abstract}
The knowledge of vascular variations, like other anatomical variations, is important during the operative, diagnostic and endovascular procedures in abdomen. This report describes variations in the celiac trunk and abnormal entry of hepatic arteries into the liver as found during routine dissection in approximately 65 years old female cadaver. The celiac trunk was two inches long and gave 2 inferior phrenic arteries, a left gastric artery, and then bifurcated into a splenic and a common hepatic artery. The common hepatic artery gave rise to left hepatic artery, which entered the liver through fissure for ligamentum venosum. The common hepatic artery then divided into right hepatic and gastroduodenal arteries. The cystic artery was a branch of the left hepatic artery. The porta hepatis of the liver was situated on the right side of the caudate lobe.

The knowledge of variation reported here is important during endovascular procedures, cholecystectomy and liver transplantation from the living donors (Fig. 2, Ref. 18). Full Text in PDF www.elis.sk

Key words: celiac trunk, inferior phrenic artery, hepatic artery, porta hepatis, liver, cystic artery, variations.
\end{abstract}

The hepatic, splenic and left gastric arteries are considered as the "main classic branches" of the celiac trunk (CT). In the embryonic life, yolk sac is supplied by vitelline arteries. Later they gradually fuse and help in formation of the arteries dorsal to the mesentery of the gut. In the adult, they are developed as CT, superior mesenteric and inferior mesenteric arteries. These vessels supply the derivatives of foregut, midgut and hindgut, respectively (1). CT supplies the parts of the foregut like liver, stomach, pancreas and superior part of duodenum. Variations of the CT and its branches, their relationship to surrounding structures are therefore of particular importance from a surgical perspective $(2,3)$.

\section{Material and methods}

During the gross anatomy dissection of the abdomen of approximately 65 year old female cadaver, we observed variations in the branching pattern, course and distribution of the celiac trunk. Some variations were also found at the porta hepatis of the liver. The celiac artery was two inches long. It first gave a pair of inferior phrenic arteries and a left gastric artery and then bifurcated into splenic and common hepatic arteries (Figs 1 and 2). The common hepatic artery gave a left hepatic artery, which entered the

${ }^{1}$ Department of Anatomy, Kasturba Medical College International Center, Manipal University, Manipal, ${ }^{2}$ Department of Anatomy, Melaka Manipal Medical College (Manipal Campus), Manipal University, Manipal, ${ }^{3}$ Department of Anatomy, Kasturba Medical College, Manipal University, Manipal, India

Address for correspondence: Satheesha Nayak B, MD, Department of Anatomy, Melaka Manipal Medical College (Manipal Campus), International Centre for Health Sciences, Manipal-576104 Karnataka, India. Phone: +91.820 .257201$ liver through the fissure for ligamentum venosum (Fig. 2). Near the inferior surface of the liver, the left hepatic artery gave a cystic artery to the gall bladder (Fig. 1). The cystic artery entered the Calot's triangle passing anterior to the common hepatic duct. The common hepatic artery terminated by dividing into gastroduodenal and right hepatic arteries. The right hepatic artery entered the liver through the porta hepatis.

The porta hepatis was situated between the caudate lobe and the right lobe of the liver. The right hepatic artery, bile duct and portal vein were seen entering/leaving the liver at porta hepatis (Fig. 2). The posterior surface of the liver was merged with the inferior surface and the groove for inferior vena cava was very small. The actual place for the vena caval groove was occupied by the porta hepatis.

\section{Discussion}

Celiac trunk is a ventral splanchnic branch of aorta. It has a short course of $1.25 \mathrm{~cm}$, after which it divides into splenic, common hepatic and left gastric arteries. Variations in the branches of the celiac trunk are common. Yuksel et al (1998) have reported a case of inferior phrenic trunk arising from celiac trunk and an aberrant right hepatic artery arising from the superior mesenteric artery. A common trunk of left gastric and left inferior phrenic arteries, arising from celiac trunk has been reported by Cavdar (1998). Bordei and Antohe (2002) have reported separate aortic origin of all the three branches of celiac trunk. They also reported the presence of celiaco-mesenteric trunk, hepatosplenic and hepatogastric trunks. Hepatogastric and hepatosplenic trunks have also been reported by Demirtas et al. (2005). Yildirim et al (2004) have reported the origin of middle colic artery from the celiac trunk. The occurrence 


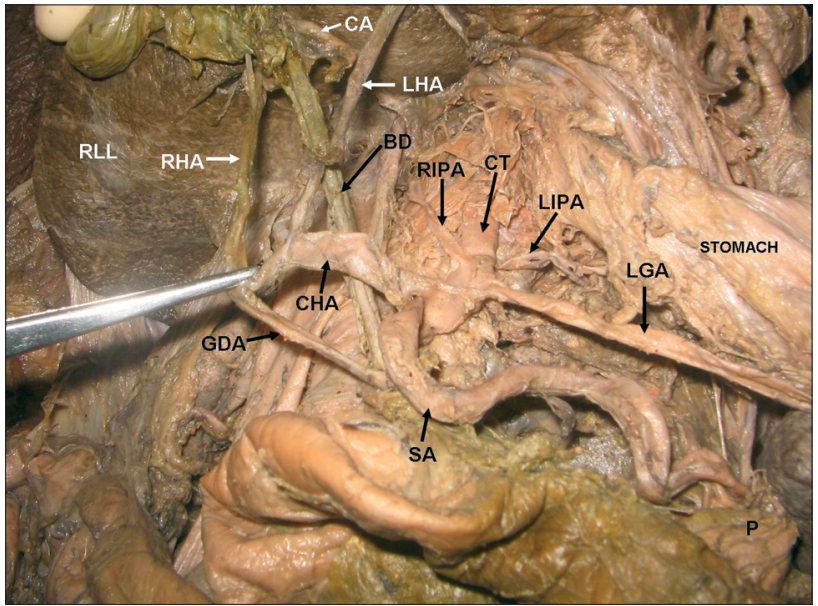

Fig. 1. Dissection demonstrating the celiac trunk and its branches. CT - celiac trunk, LIPA - left inferior phrenic artery, RIPA - right inferior phrenic artery, CHA - common hepatic artery, RHA - right hepatic artery, LHA - A left hepatic artery, CA-cystic artery, GDAgastroduodenal artery, SA - splenic artery, LGA - left gastric artery, BD - bile duct, RLL - right lobe of liver.

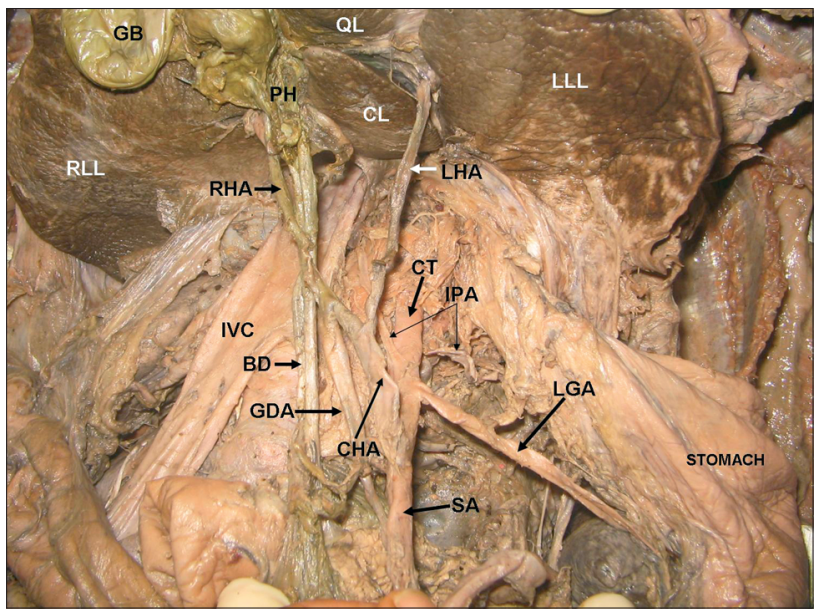

Fig. 2. Dissection demonstrating the branches of celiac trunk and entry of hepatic arteries into the liver. CT - celiac trunk, IPA - left inferior phrenic arteries, CHA - common hepatic artery, RHA - right hepatic artery, LHA - A left hepatic artery, GDA - gastroduodenal artery, SA - splenic artery, LGA - left gastric artery, BD - bile duct, RLL - right lobe of liver, $\mathbf{L L L}$ - left lobe of liver, $\mathrm{CL}$ - caudate lobe, $\mathrm{QL}$ - quadrate lobe, GB - gall bladder, $\mathrm{PH}$ - porta hepatis, IVC - inferior vena cava.

of a celiacomesenteric trunk is only $1-2.7 \%$ of cases (Cavdar et al, 1997). Cicekcibasi et al (2005) have reported the presence of a celiacomesenteric trunk, which gave rise to the left gastric, the common hepatic, the splenic, the left gastro-epiploic, the right and left inferior phrenic arteries. Nayak (2006) has reported the presence of an abnormally long celiaco-mesenterico-phrenic trunk, which gave a common inferior phrenic trunk, a celiac trunk and a superior mesenteric artery. The celiac trunk is usually a short trunk. In the current case, the trunk was abnormally long and branched in a different manner than that available in the literature.
Variations in the origin, course and distribution of the hepatic arteries is common and are surgically important. Abdullah et al (2006), have done an extensive study on the variations of the hepatic arteries in liver transplant cases. In their study on 932 patients, normal hepatic artery distribution was found in $68.1 \%$ cases. Variations of hepatic artery were detected in $31.9 \%$ and were divided into three groups describing 48 common hepatic artery anomalies, 236 left or right hepatic artery anomalies and 13 rare variations including one case of right hepatic artery stemmed from the inferior mesenteric artery and one case of normal common hepatic artery passed behind the portal vein. Uva et al (2007) reported a common hepatic artery arising from the left gastric artery in a cadaveric liver donor.

The origin of the left hepatic artery directly from the common hepatic artery and giving a large branch to the gall bladder and then entering the liver through fissure for ligamentum venosum is interesting and has not been reported earlier. This kind of abnormal course and distribution may be useful for surgeons doing cholecystectomy and liver transplant in living donors.

The cystic artery is normally a branch of right hepatic artery. It reaches the neck of the gall bladder by passing posterior to the common hepatic duct. Variations in the origin of the cystic artery are known. Mlakar et al (2003) studied the blood supply of gall bladder in 81 subjects. The gallbladder was supplied by one cystic artery in $86 \%$ and by two arteries in $14 \%$ of cases. When a single artery was present, it originated from the right hepatic artery in $53 \%$ of livers. In the study of Flisinski et al (2004) the cystic artery was most often $(97.06 \%)$ a single vessel and rarely $(2.94 \%)$ a double vessel. It arose most often $(82.34 \%)$ from the right proper hepatic artery, rarely from its trunk $(8.82 \%)$ or its left branch $(5.88 \%)$ and most rarely (2.94\%) from the gastroduodenal artery. A thorough knowledge about the origin of the cystic artery is surgically important, especially when intraoperative or post-operative bleeding occurs in the gallbladder fossa. Insufficient recognition of its anatomical variation may contribute to a dangerous situation, especially during laparoscopic cholecystectomy. In the present case, origin of the cystic artery from abnormal left hepatic artery and its course anterior to the common hepatic duct is noteworthy.

Inferior phrenic arteries normally arise from the abdominal aorta just below the aortic opening of the diaphragm. Abnormal origins of these arteries also exist, including origin from the celiac trunk (Yuksel and Sargon, 1992; Nakamura et al, 2003). Loukas et al (2005) have examined 300 normal cadavers for variations of the inferior phrenic artery. According to their observations, the inferior phrenic artery originated from the: a) celiac trunk in $40 \%$ of the specimens; b) aorta in $38 \%$; c) renal in $17 \%$; d) left gastric in $3 \%$; and e) hepatic artery proper in $2 \%$ of the specimens. The left inferior phrenic artery originated from the: a) celiac trunk in $47 \%$; b) aorta in $45 \%$; c) renal in $5 \%$; d) left gastric in $2 \%$; and e) hepatic artery proper in $1 \%$ of the specimens. The right inferior phrenic artery is always associated with hepatocellular carcinoma and serves as the major collateral artery adjunct to the hepatic artery. Knowledge of variations of the inferior phrenic artery is very important in the surgeries of suprarenal glands and also the surgeries involving diaphragm. 
$120-122$

The porta hepatis is the vascular hilum of the liver and it is normally situated between the caudate and quadrate lobes. In our case, the porta hepatis was found mainly between the caudate lobe and right lobe of the liver, where the hepatic ducts were coming out and branches of portal vein and right hepatic artery were entering the liver. The groove for inferior vena cava was shifted to a much higher level. This kind of a shift of the porta hepatis might lead to confusions to the surgeons during gall bladder surgeries or liver transplant surgeries.

\section{References}

1. Sadler TW. Langman's Medical Embryology. 10th Ed., Baltimore: Williams and Wilkins, 2006; 183.

2. Vandamme JP, Bonte J. The branches of the celiac trunk. Acta Anat 1985; 122: 110-114.

3. Kahraman G, Marur T, Tanyeli E, Yıldırım M. Hepatomesenteric trunk. Surg. Radiol Anat 2001; 23: 433-435.

4. Yuksel M, Yalin A, Weinfeld AB. Concurrent anomalies of the abdominal arteries: an extremely long coeliac trunk, an inferior phrenic trunk, and an aberrant right hepatic artery. Kaibogaku Zasshi 1998; 73 (5): 497-503.

5. Cavdar S, Sehirli U, Pekin B. Celiacomesenteric trunk. Clin Anat 997; 10 (4): 231-234.

6. Bordei P, Antohe DS. Variations of the celiac trunk branches in the fetus. Morphologie 1997; 86 (274): 43.

7. Demirtas K, Gulekon N, Kurkcuoglu A, Yildirim A, Gozil R. Rare variation of the celiac trunk and related review. Saudi Med J 2005; 26 (11): 1809-1811.

8. Yildirim M, Celik HH, Yildiz Z, Tatar I, Aldur MM. The middle colic artery originating from the coeliac trunk. Folia Morphol (Warsz) 2004; 63 (3): 363-365.
9. Cavdar S, Gurbuz J, Zeybek A, Sehirli U, Abik L, Ozdogmus O. A variation of coeliac trunk. Kaibogaku Zasshi 1998; 73 (5): 505-508.

10. Cicekcibasi AE, Uysal II, Seker M, Tuncer I, Buyukmumcu M, Salbacak A. A rare variation of the coeliac trunk. Ann Anat 2005; 187 (4): 387-391.

11. Nayak S. Common celiaco-mesenterico-phrenic trunk and renal vascular variations. Saudi Med J 2006; 27 (12): 1894-1896.

12. Abdullah SS, Mabrut JY, Garbit V, De La Roche E, Olagne E, Rode A, Morin A, Berthezene Y, Baulieux J, Ducerf C. Anatomical variations of the hepatic artery: study of 932 cases in liver transplantation. Surg Radiol Anat 2006; 28 (5): 468-473.

13. Uva P, Arvelakis A, Rodriguez-Laiz G, Lerner S, Emre S, Gondolesi G. Common hepatic artery arising from the left gastric artery: a rare anatomic variation identified on a cadaveric liver donor. Surg Radiol Anat 2007; 29 (1): 93-95.

14. Mlakar B, Gadzijev EM, Ravnik D, Hribernik M. Anatomical variations of the cystic artery. Eur J Morphol 2003; 41 (1): 31-34.

15. Flisinski P, Szpinda M, Flisinski M. The cystic artery in human fetuses. Folia Morphol (Warsz) 2004; 63 (1): 47-50.

16. Yuksel M, Sargon M. A variation of a coeliac trunk. Okajimas Folia Anat Jpn 1992; 69: 173-175.

17. Nakamura Y, Miyaki T, Hayashi S, Iimura A, Itoh M. Three cases of the gastrosplenic and the hepatomesenteric trunks. Okajimas Folia Anat Jpn 2003; 80: 71-76.

18. Loukas M, Hullett $\mathbf{J}$, Wagner T. Clinical anatomy of the inferior phrenic artery. Clin Anat 2005; 18 (5): 357-365.

Received January 31, 2010. Accepted December 18, 2011. 